\title{
INVERSE DESIGN OF NACELLES USING MULTI-BLOCK NAVIER STOKES CODES
}

D. A. Naik

ViGYAN, Inc., Hampton, Virginia 23666

S. E. Krist, R. L. Campbell, and V. N. Vatsa

NASA Langley Research Center, Hampton, Virginia 23681

P. G. Buning

NASA Ames Research Center, Moffett Field, California 94035

and

L. M. Gea

McDonnell Douglas Aerospace, Long Beach, California 90810

\begin{abstract}
The objective of this work is to reshape a nacelle to achieve a specified nacelle pressure distribution. The nacelle may be either isolated or installed on an airplane. There are no restrictions on the attitude (toe, incidence, and roll) and position of the nacelle. The design algorithm is coupled to two different multi-block 3-D Navier Stokes flow solvers. The coupling between design and analysis is automated to the point where the design proceeds with minimal user input.

\section{Nomenclature}

C - Local nacelle or wing chord.

CDISC - Constrained DISC.

$\mathrm{Cp}$ - Coefficient of pressure.

DISC - Direct Iteration Surface Curvature.

$\mathrm{O}(.$.$) - Order of magnitude.$

$\mathrm{R}$ - Local nacelle radius.

$\mathrm{X}$ - Streamwise coordinate.

theta - Angle running from the nacelle crown through the keel (outboard side) and back up to the crown (inboard side).
\end{abstract}

\section{Introduction}

Given the harsh reality of placing capital intensive products in the modern marketplace, it is an ongoing quest to improve the operational efficiency and cost effectiveness of any commercial airplane. The emergence of the current generation of large turbofans, in the 80,000 to 100,000 pound thrust class, presents anew the opportunity to optimize or reduce the drag associated with the outer surface of the engine nacelle. These large nacelles could account for a good portion of airplane drag.

The problem of external cowl design may be broadly placed in two separate, yet complementary categories: (1) the design of an isolated cowl immersed in a uniform flow; and, (2) the design of an installed cowl with the attendant non-uniform flow. The former should enable the designer to derive a cowl geometry starting from a fairly rudimentary first guess. The resulting design will be inherently symmetrical about the vertical plane, thus allowing for a universal nacelle that can be installed on either side of the airplane. On the other hand, the inverse design of an installed nacelle will always result in a nacelle that is non-symmetric about the vertical plane, unless the designer can invoke special options to force symmetry. 
Thus, a tool for the inverse design of nacelles must be flexible enough for a designer to be able to produce an initial design, then study changes required to the proposed design because of the presence of the airplane flowfield, and finally, perform trade studies of the pros and cons of nacelle symmetry. For example, the cruise benefit gained from achieving laminar flow over a non-symmetric nacelle might be significant enough to outweigh other issues.

\section{Method}

The design method used in the codes is the CDISC inverse design algorithm1. CDISC is an extension of the basic DISC method of Campbell2\&3. At the very crux, DISC is a pressure based design method in that the change in surface geometry is related to a change in pressure. The user starts with an initial "rough" cut of the geometry. The initial geometry is gridded and then analyzed using a CFD code. The surface pressure is then examined and a target pressure distribution is specified which is an improvement to the original pressure distribution. The difference between the desired pressure and the existing pressure is used to compute a change in geometry. The volume grid is automatically adjusted to accommodate the new geometry and a fresh analysis is performed. The results from this analysis are then compared to the target and a new geometry is computed and analyzed. The process continues until the user is satisfied with the analysis pressure.

The nature of the relationship between geometry and pressure is different for subsonic (where change in pressure is related to change in curvature) and supersonic (where change in pressure is related to change in slope) flow but the basic idea is the same for both: that the resultant change in geometry depends on the desired change in pressure. This kind of design philosophy is ideally suited for nacelle design because nacelle flows are largely attached. Without special treatments, one would expect DISC to fail in regions of massive separation because the streamline curvature relationship would no longer be valid on the surface.

In CDISC, one specifies desirable characteristics of the target pressure distribution. For example, one might specify pressure gradients in one region, shock position in another region, pressure levels in a third and then smooth selectively certain critical regions of the design space. These regions can be both chordwise and spanwise (circumferential) in extent. The target file is automatically generated and updated at each step based on all these constraints. One can also specify geometry constraints such as minimum thickness and leading edge radius.

Additionally, CDISC has some global constraints for quantities like twist distribution, lift coefficient and pitching moment that are especially useful for the design of wings. In the current version of CDISC an attempt is made to satisfy all constraints by iterating through them a set number of times in the order in which they are listed.

\section{Implementation}

DISC and CDISC have been coupled to many CFD codes. Airplane winglets and installed nacelles have been designed using DISC coupled to an Euler code4\&5. DISC has been used with the CFL3D Navier-Stokes code for the design of isolated nacelles6.

The coupling of CDISC with the multi-block codes, TLNS3D-MB (Thin-Layer Navier-Stokes 3-Dimensional Multi-Block from Vatsa7,8\&9) and OVERFLOW (a 3-D Navier Stokes code for overset Grids from Buning10-13) is presented here. These two implementations of CDISC allow for the design of either isolated or installed nacelles with any combination of toe, inclination and roll angle for the 
nacelle. While this paper concentrates on nacelle design, it is important to note that the implementations are sufficiently general to allow for the redesign of any other part of the airplane.

\section{TLNS3D-MB}

CDISC is incorporated as a system of subroutines called by the main TLNS3D-MB driver. The CDISC routines are only called when the residual has dropped to a value chosen by the user. Once called, CDISC first extracts all relevant volume grids for the cowl surface from the grid array. These volume grids are then oriented to place the nacelle centerline on the x-axis. The volume grid is now on a sort of "virtual" workbench on which the design changes for the current design cycle can be easily performed.

At each chosen streamwise design station, positioned circumferentially around the nacelle, the geometry is changed to offset the discrepancy between the analysis and the target pressures. The portions of the nacelle between target stations are changed in a linear fashion. Finally, circumferential smoothing is applied as desired. The volume grids are then perturbed to accommodate this new nacelle surface. Finally, the new grids are restored to their proper orientation and are placed back into the grid array for further analysis. This new analysis proceeds until a user specified drop in the residual between design iterations is achieved, after which a new design cycle is begun.

The TLNS3D-MB solver, used herein, incorporates mesh sequencing and multigrid, but requires point-matched grids. The code allows a choice of turbulence models. The Spalart-Allmaras one-equation model14 was used for the examples in this paper.

One limitation in the above approach is the difficulty in constructing point-matched multi-block grids for complex configurations. For this reason, the overset grid approach is more attractive although there might be reservations among some in the aerospace community regarding its accuracy, particularly in regard to flux conservation. It should be noted, however, that most patched grid multi-block codes use non-conservative interpolation for block interfaces as well.

\section{OVERFLOW}

Except for the process of monitoring when to start and stop analysis and design, the entire CDISC system is largely run as a post-processor outside OVERFLOW. Control passes out of OVERFLOW whenever a user specified criterion (typically, the residual drop on the grid involving the component to be designed) is reached. Once out of OVERFLOW, the volume grid and restart files are used to compute the pressure on the fan cowl. The relevant grids are then oriented on to the "virtual" workbench described above and the CDISC geometry modifier is invoked. The grid is then automatically adjusted to accommodate the geometry change. The cowl grids are then restored to their proper orientation.

Next, all relevant grids are re-projected and recomputed. For example, the pylon might have to be reprojected back onto the new nacelle surface. Next the pylon grid will have to be restretched and the nacelle fringe points re-projected back onto the pylon grid. Finally, the new grid system must be run through the mesh interpolation program, PEGSUS15, in order to update the interpolation stencils between the various grids, before OVERFLOW can be restarted for analysis of the new geometry. The analysis continues until the next residual drop is achieved.

The OVERFLOW code also allows a choice of turbulence models. The Baldwin-Barth model16 was used for the examples given in this paper.

\section{Results}




\section{Isolated nacelle}

An example of designing an isolated nacelle using TLNS3D-MB is shown in Figures 1 - 4. The geometry is an isolated fan-cowl and core cowl. The grid for the fan-cowl and core-cowl comprised six blocks containing a total of one million grid points. The core cowl was run inviscid while the fan cowl was run viscous. The external surface of the fan cowl was redesigned.

Figure 1 shows the convergence history for the design of this isolated nacelle. The spikes indicate the design iterations. The horizontal axis on the plot is a measure of the computational work that was performed. It is readily seen that in order to achieve the same $\mathrm{O}(3.5)$ drop in residual, the design takes roughly 420/290 1.45 times the computational work of an analysis of a fixed geometry. Similar run comparisons were observed with the OVERFLOW runs.

Figure 2 shows that the target coefficient of pressure has been closely matched after eight design cycles. The target stations were grid lines closest to the 45 degrees, 90 degrees, 135 degrees, 180 degrees, 225 degrees, 270 degrees and 315 degrees cuts respectively. The run was set up so that no design changes were allowed on the upper part of the nacelle between 340 degrees and 20 degrees. One can see that the final $\mathrm{Cp}$ distribution is quite different from the initial distribution.

This case is a good check-out run because the target pressure distribution was deliberately obtained from a geometry that was known a priori. While there is no guarantee that the inverse design will produce this identical geometry, Figures 3 and 4 show that the designed geometry is quite close to the "target" geometry. The dotted lines for the design shape are almost completely masked by the solid lines for the known "target" geometry. Note also that the resultant nacelle is symmetrical about the vertical plane. This symmetry condition was not forced during the design by using the CDISC option of aliasing corresponding left side stations to those on the right. Rather, the symmetry of the original geometry was maintained because of the uniform inflow condition. Note also that the original and designed nacelles are not symmetrical, about a horizontal plane passing through the nacelle centerline, because of the inlet droop.

This example shows that a desired nacelle can be obtained from a radically different initial profile. The technique is directly applicable to any multi-block grid containing an installed nacelle.

\section{Installed Nacelle}

The nacelle designed above was installed on a transport airplane operating at a mid-cruise Mach number of 0.85 . This flow-through nacelle was installed with a toe-in of 1.5 degrees and an upward tilt of 2 degrees. The cruise angle of attack was roughly 1.5 degrees which meant that the installed nacelle faced an inflow stream at a much higher effective angle of attack than in the example above. Also the inflow was non-uniform because of the presence of the airplane wing and fuselage.

This redesign was performed with OVERFLOW-CDISC. The fuselage-wing, pylon, fan-cowl, core-cowl, bifurcator, pylon shelf and far-field are represented by seven overset grids. The grid topology is not presented here because it is very similar to that described in reference 10 which also shows various views of the overset grid system. During each design cycle, the fan-cowl grid was the only grid extracted to the virtual workbench. The design was carried out over the entire circumference of the fan cowl.

Figure 5 is a plot of the convergence history. The nine spikes indicate the design iterations. An erroneous tenth spike is also visible. This spike may be ignored; it occurred because of a malfunction in 
the computer system. Analysis of the final design change was stopped after $\mathrm{O}(3.5)$ convergence was achieved.

Figure 6 shows the $\mathrm{Cp}$ and geometry history for the nine design cycles at an inboard cut, the keel line, and an outboard cut. In this example, left-right symmetry is not maintained. The design is an attempt to achieve a favourable pressure gradient over the first 50 percent of the nacelle chord, while still allowing the inboard side of the nacelle to have overall lower $\mathrm{Cp}$ levels than the outboard side. This concession is not necessary, but is typical of the Mach suppression effect seen on the inboard side of an installed nacelle17. With CDISC one was able to achieve the characteristics of a desired pressure distribution without compromising the levels of the existing analysis pressure.

Figure 7 is representative of the wing $\mathrm{Cp}$, which was largely unaffected by the design changes to the nacelle. Although not shown, the wing drag did not change despite the dramatic changes in nacelle $\mathrm{Cp}$.

\section{Conclusions}

Tools have been developed for the inverse design of a component of a complete airplane configuration while it is in the presence and under the influence of the rest of the airplane. Except for the initial grid generation, which will depend on the particular problem of interest, the design process is automated.

The results showed the specific application of these tools to the design of turbofan nacelles. Additionally, it was shown that computational cost of the design is of the same order as that of a comparable analysis.

\section{Acknowledgments}

A part of this research is funded by the National Aeronautics and Space Administration, Langley Research Center, Component Integration Branch, under NASA Contract No. NAS1-19672.

Computational support was provided by the National Aerodynamic Simulation program. The technical advice of Dr. Richard D. Cedar from General Electric Aircraft Engines is gratefully acknowledged.

\section{References}

1. Campbell, R. L.: "An Approach to Constrained Aerodynamic Design with Application to Airfoils," NASA-TP-3260, November 1992.

2. Yu, N. J., and Campbell, R. L.: "Transonic Airfoil and Wing Design using Navier-Stokes Codes," AIAA CP-92-2651.

3. Smith, L. A., and Campbell, R. L.: "Applications of a Direct/Iterative Design Method to Complex Transonic Configurations," NASA-TP-3234, September 1992.

4. Lin, W. F., Chen, A. W., and, Tinoco, E. N.: "3D Transonic Nacelle and Winglet Design," AIAA CP-90-3064.

5. Chen, H. C., Su, T. Y., and Kao, T. J.: "An Installed Nacelle Design Code Using a Multiblock Euler Solver, Volume I: Theory Document," NASA CR 189652, September 1992.

6. Bell, R. A., and Cedar, R. D.: "An Inverse Method for the Aerodynamic Design of Three-Dimensional Aircraft Engine Nacelles," Third International Conference on Inverse Design Concepts and Optimization in Engineering Sciences," October 23-25, 1991. 
7. Vatsa, V. N., Sanetrik, M. D., and Parlette, E. B.: "Development of a Flexible and Efficient Multigrid-Based Multi-block Flow Solver," AIAA CP-93-0677, January 1993.

8. Vatsa, V. N., and Wedan, B. W.: "Development of an Efficient Multigrid Code for 3-D Navier-Stokes Equations," AIAA CP-89-1791.

9. Vatsa, V. N., Wedan, B. W., Turkel, E.: "3-D Euler and Navier-Stokes Calculations for Aircraft Components," Transonic Symposium: Theory, Application, and Experiment," NASA CP-3020, Volume 1, Part 2, pp. 571-579, April 1988.

10. Gea, L. M., Halsey, N. D., Intemann, G. A., and Buning, P. G.: "Applications of the 3-D Navier-Stokes Code OVERFLOW for Analyzing Propulsion-Airframe Integration Related Issues on Subsonic Transports," ICAS Paper 94-3.7.4, 19th Congress of the International Council of the Aeronautical Sciences, Anaheim, California, September 18-23, 1994.

11. Buning, P. G., Parks, S. J., Chan, W. M., and Renze, K. J.: "Application of the Chimera overlapped grid scheme to simulation of Space Shuttle ascent flows," Presented at the 4th International Symposium on Computational Fluid Dynamics, Davis, CA, September 9-12, 1991

12. Renze, K. J., Buning, P. G., and Rajagopalan, R. G.: "A comparative study of turbulence models for overset grids," AIAA CP-92-0437, January 1992.

13. Parks, S. J., Buning, P. G., Chan, W. M., and Steger, J. L.: "Collar grids for intersecting geometric components within the Chimera overlapped grid scheme," AIAA CP-91-1587, June 1991.

14. Spalart, P. R., and Allmaras, S. R.: "A One-Equation Turbulence Model for Aerodynamic Flows," AIAA CP-92-0439, January 1992.

15. Suhs, N. E., and Tramel, R. W.,: "PEGSUS 4.0 User's Manual," AEDC-TR-91-8, November 1991.

16. Baldwin, B. S., and Barth, T. J.: "A One-Equation Turbulence Model for High Reynolds Number Wall-Bounded Aerodynamic Flows," NASA TM-102847, August 1990.

17. Ostrander, M. J., and, Cedar, R. D., "Analysis of High Bypass Ratio Engine Installation Using the Chimera Domain Decomposition Technique," AIAA CP-93-1808, June 1993. 


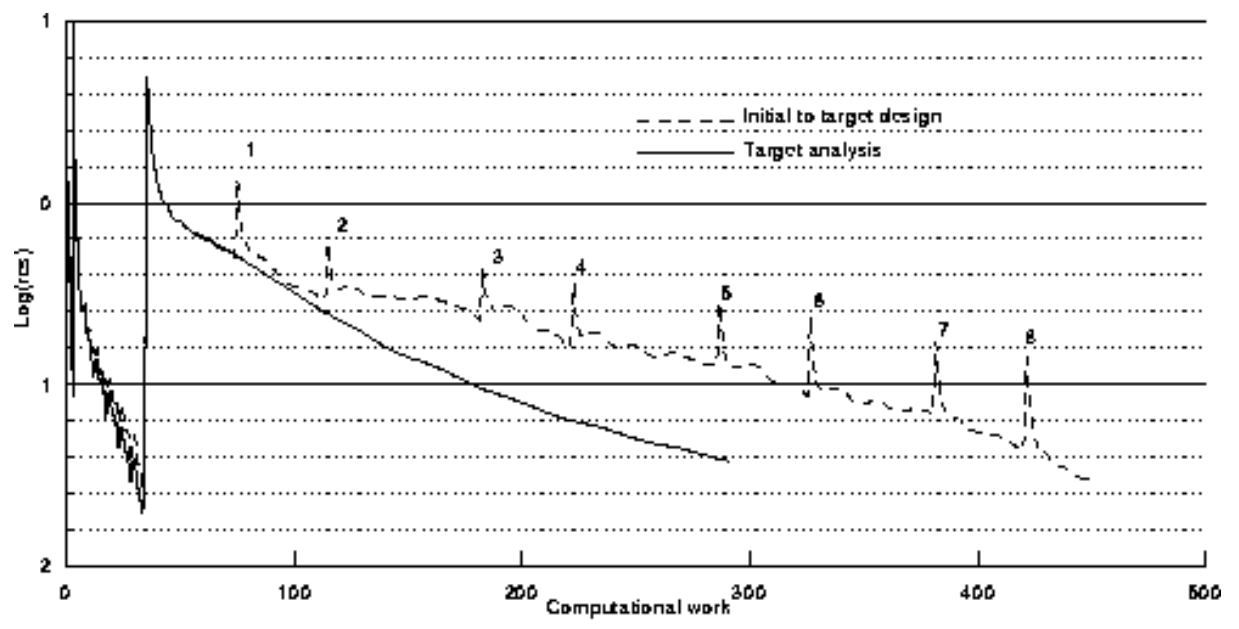

Fig. 1 Conuergenee history for inu crse design of isloated racelle using TLNS3D NB DISC. The peak residual (nok yis ible in the pot) was 2.05 on the coars cstmeash. Two hurdred cycles of mesh s cquencing were performed on two coarser lev ds before thres level multigrid was s arted.

$\mathrm{CP}_{\mathrm{p}}$

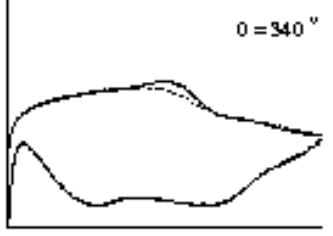

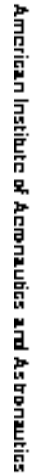

$\mathrm{Cp}_{\mathrm{p}}$

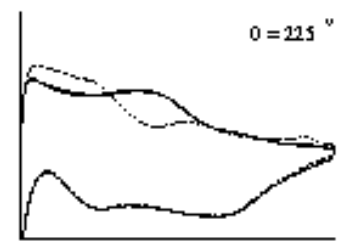

XIC

$0=270^{\circ}$

$\mathrm{Cp}$

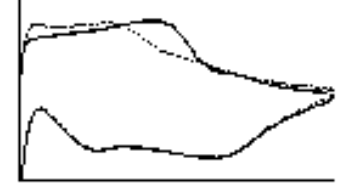

.
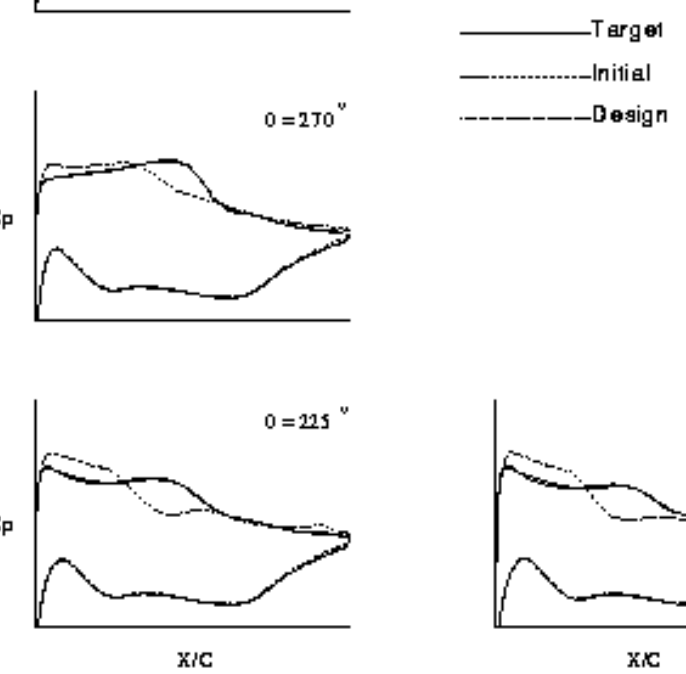

XiC

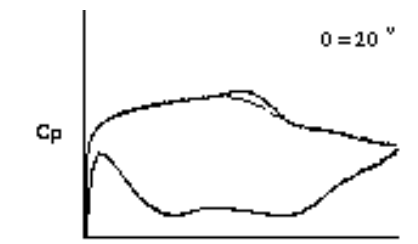

$\mathrm{Cp}$
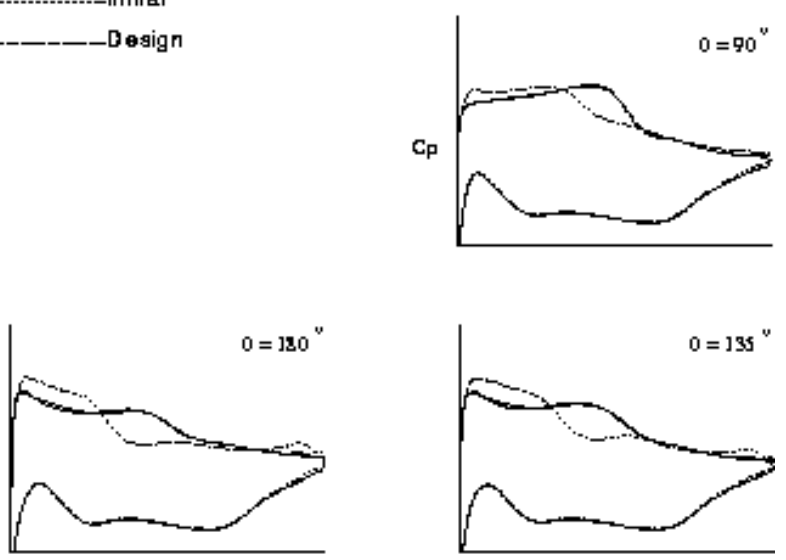

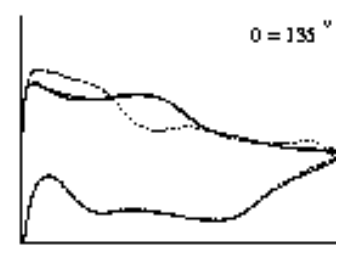

XiC

Fig 2 Targct, initial and design Cp for isolated nacelle. After eight design cy cles. 


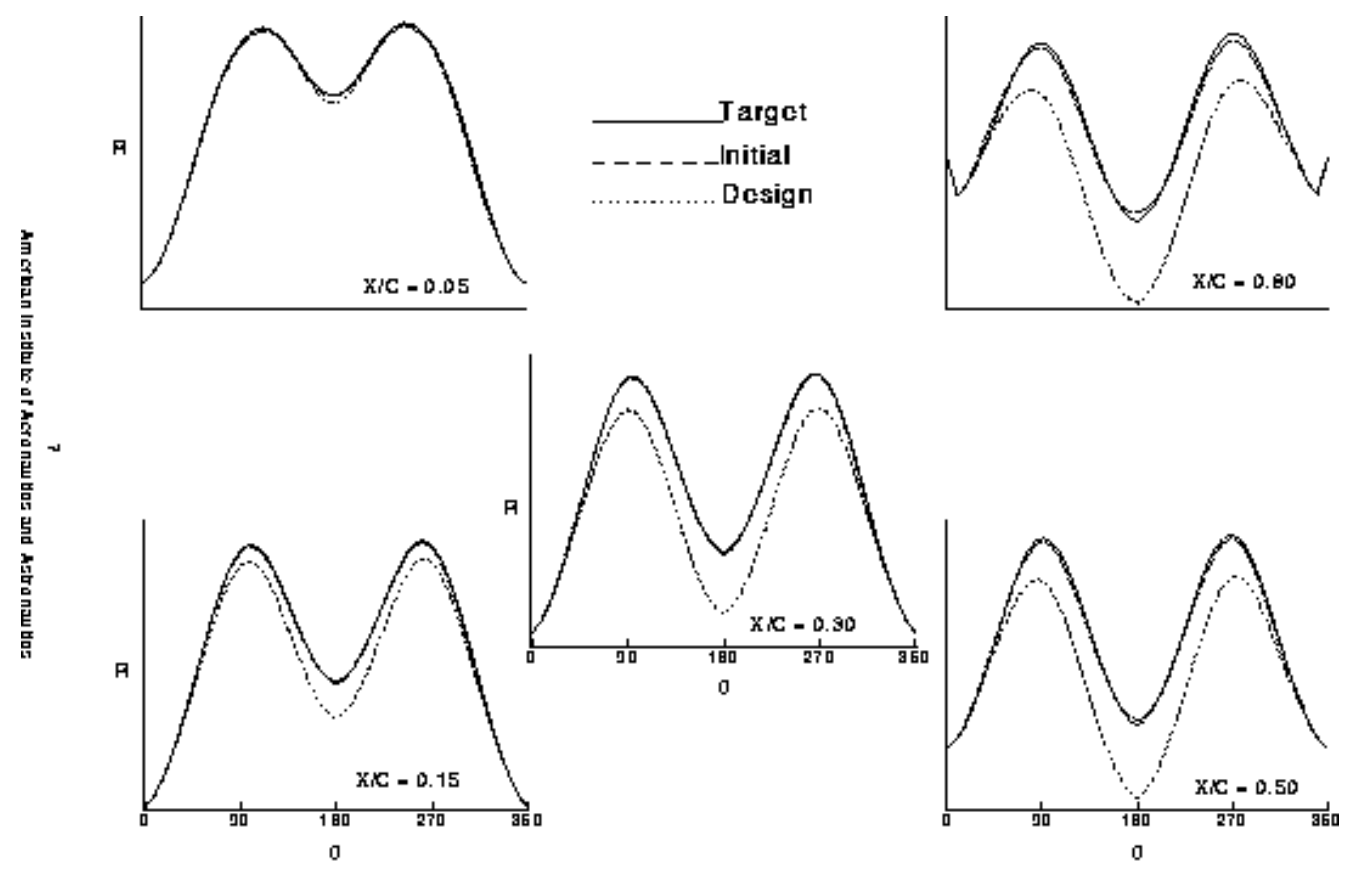

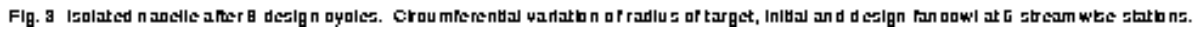

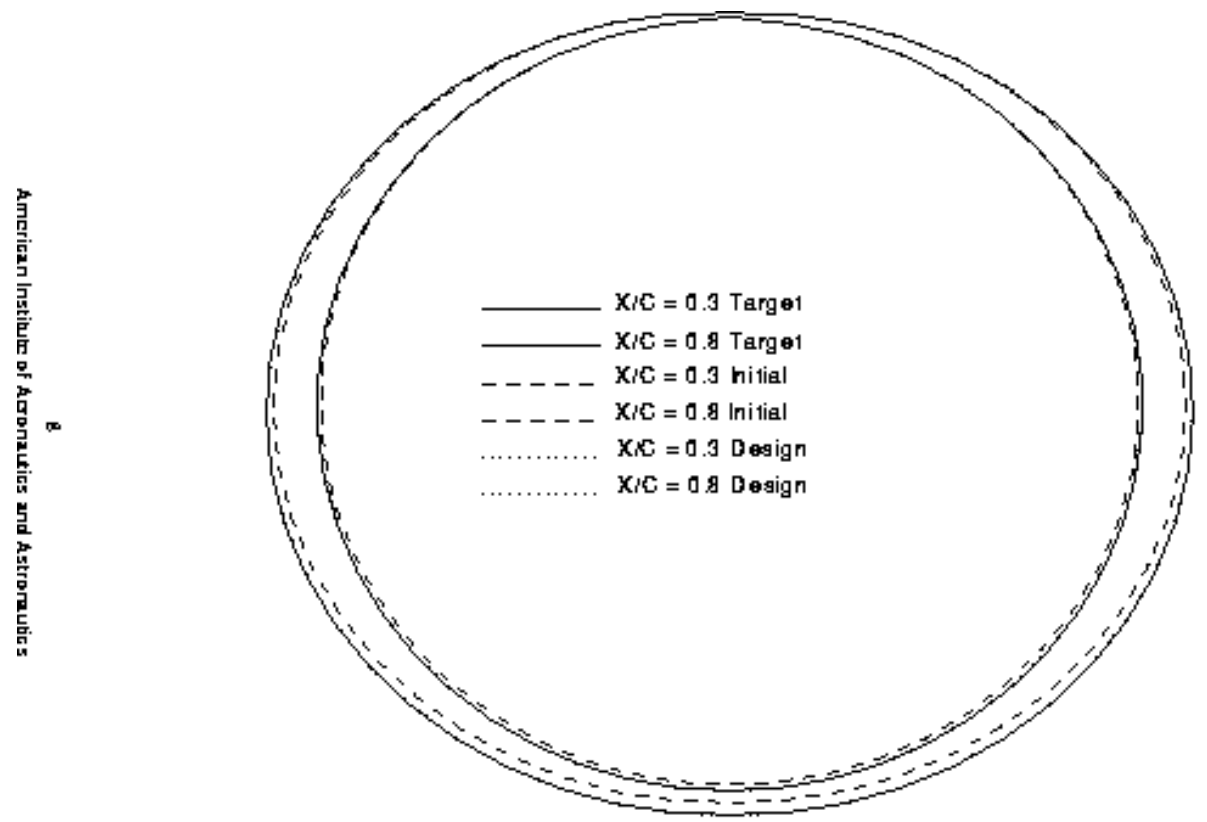

Fig. 4 lsolated nacelle after $B$ design cycks. Cross sectional wiew of targct, initial and design fancowl at two streamwise stations. 

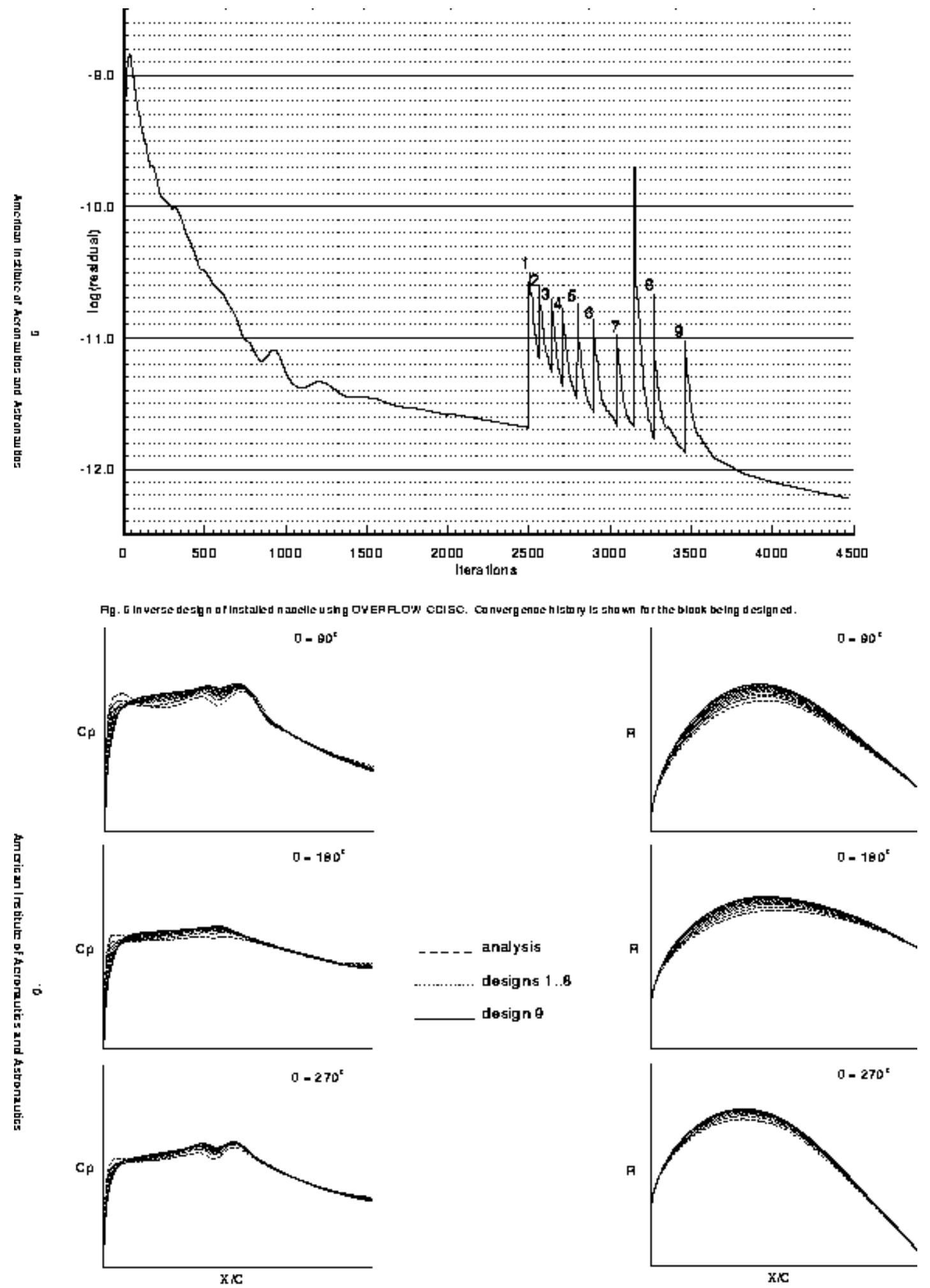

Fig. \& CP and radius history for thres tuts on an installed nar clle that was inverse des igned with CVERFLCW CDISC. 


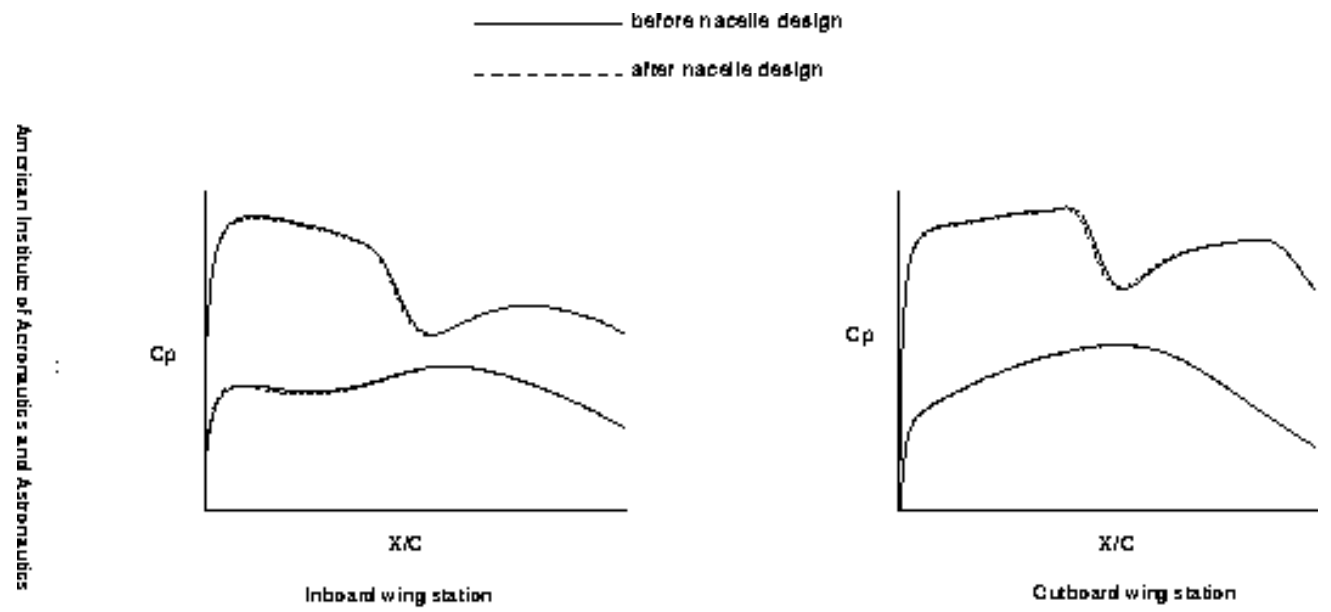

Fig. 7 Effect of inuerse design of installed nacelle on wing section Cp. CWERFLCW CDISC resut

Copyright (c) 1995 by Dinesh A. Naik. Published by the American Institute of Aeronautics and Astronautics, Inc. with permission. 